\title{
APLICANDO A SOFT SYSTEMS METHODOLOGY A UM PROJETO DE EXTENSÃO UNIVERSITÁRIA
}

\author{
Daniel Braga Arêas \\ Centro Universitário Geraldo di Biase \\ Marcos Estellita Lins \\ Federal University of Rio de Janeiro
}

\begin{abstract}
RESUMO
Esse trabalho exemplifica uma aplicação da Soft Systems Methodology (SSM) a um projeto de extensão universitária. Mais especificamente, serão aplicadas as etapas de descrição da definição raiz e de construção de modelos de sistemas relevantes (fases três e quatro, respectivamente, do modelo de sete estágios da SSM). Para isso será utilizado como estudo de caso o projeto PAPESCA (Pesquisa-ação na Cadeia Produtiva da Pesca), coordenado pelo Núcleo de Solidariedade Técnica do Departamento de Engenharia Industrial da Universidade Federal do Rio de Janeiro (SOLTEC/UFRJ). O objetivo é mostrar como a Soft Systems Methodology pode ser útil ao levantar e explorar diversas perspectivas do objeto de estudo, funcionando assim como um indutor do debate em torno de possíveis melhorias, e auxiliar na organização do projeto ao estruturar as atividades necessárias para a consecução de seus objetivos.
\end{abstract}

Palavras-Chave: Extensão Universitária, Soft Systems Methodology, Pesquisa Operacional Soft

\begin{abstract}
This paper illustrates an application of Soft Systems Methodology (SSM) at a university extension project. More specifically it will be applied the phases of description of the root definition and construction of relevant systems models (phases three and four, respectively, of the seven-stage model of SSM). It will be used as a case study the project PAPESCA (Pesquisa-ação na Cadeia Produtiva da Pesca) which is coordinated by the Núcleo de Solidariedade Técnica of the Industrial Engineering Department of Federal University of Rio de Janeiro (SOLTEC/UFRJ). The aim is to show how the Soft Systems Methodology can be useful to raise and explore various perspectives of the study object and thus act as an inducer of the debate on possible improvements, and assist the project's organization by structuring the activities necessary to achieve its objectives.
\end{abstract}

Key words: University Extension, Soft Systems Methodology, Soft Operational Research 


\section{INTRODUÇÃO}

É notável o desenvolvimento da extensão universitária no Brasil, nas últimas décadas. Das primeiras iniciativas isoladas no início do Século XX, ainda de caráter assistencialista, até o atual reconhecimento da sua indissociabilidade do ensino e da pesquisa (expressa na Constituição de 1988) e o caráter dialógico da suas ações (segundo as diretrizes do Fórum de Pró-Reitores de Extensão das Universidades Públicas Brasileiras FORPROEX), a extensão universitária vem se estabelecendo como pilar fundamental da educação no Brasil.

O fortalecimento da sociedade civil nos anos 80 ensejou o surgimento de uma nova forma de se pensar a relação Universidade-sociedade, e a redefinição do próprio conceito de Extensão Universitária. Dois momentos foram marcantes nesse processo: a criação do Fórum de Pró-Reitores de Extensão das Universidades Públicas Brasileiras (FORPROEX) em 1987 e a publicação do Plano Nacional de Extensão, publicado em novembro de 1999. O Plano Nacional de Extensão define a extensão como parte indispensável do pensar e fazer universitários (PLANO NACIONAL DE EXTENSÃO UNIVERSITÁRIA, 1999).

THIOLLENT (2008) ressalta também que, em paralelo à expansão da atividade de extensão universitária, observa-se um significativo avanço no que se refere às metodologias empregadas. Muitos programas e projetos de extensão são planejados e executados com metodologias bem definidas, e em especial, destaca-se o crescente uso de métodos participativos, em especial a pesquisa-ação. As características interativas, comunicativas e/ou participativas inerentes à atividade de extensão favorecem o uso de tais métodos.

Os Métodos de Estruturação de Problemas (Problem Structuring Methods ROSENHEAD, 1996) da Pesquisa Operacional Soft podem ser muitos úteis no contexto das tendências atuais da extensão universitária no Brasil, pois se caracterizam por uma ampla participação dos atores interessados (cujos interesses e pontos de vista sobre a situação em estudo podem divergir) e pela incorporação dos aspectos humanos nos processos de modelagem dos problemas e tomada de decisão. Além disso, tais métodos apresentam várias características filosóficas e metodológicas semelhantes às da pesquisa-ação.

Assim, esse artigo exemplifica uma aplicação da Soft Systems Methodology (SSM), uma das mais utilizadas abordagens da PO-Soft, a um projeto de extensão universitária. Mais especificamente, serão aplicadas as etapas de descrição da definição raiz e de construção de modelos de sistemas relevantes (fases três e quatro, respectivamente, do modelo de sete estágios da SSM). Para isso será utilizado como estudo de caso o projeto PAPESCA (Pesquisa-ação na Cadeia Produtiva da Pesca), desenvolvido pelo Núcleo de Solidariedade Técnica da Escola Politécnica da UFRJ (SOLTEC/UFRJ).

\section{A SOFT SYSTEMS METHODOLOGY}

A Soft Systems Methodology (SSM) é uma metodologia pertencente à vertente conhecida como Pesquisa Operacional Soft (ROSENHEAD e MINGERS, 2001) e voltada para o estudo, exploração e apoio à tomada de decisão em "situações-problema" organizacionais. A SSM foi desenvolvida por Peter Checkland e seus colegas do Departamento de Engenharia de Sistemas da Universidade de Lancaster, a partir de uma série de projetos de pesquisa-ação realizados em organizações públicas e privadas. O objetivo da pesquisa era observar os resultados da aplicação do pensamento sistêmico clássico a problemas do "mundo real" que envolvessem aspectos sociais e humanos. O desenvolvimento (tanto teórico quanto prático) da SSM está registrado em uma série de livros e artigos (CHECKLAND, 1972, 1981, 1999; CHECKLAND e SCHOLES, 1990; CHECKLAND e POULTER, 1994, 2006).

A SSM emerge a partir da dificuldade de se aplicar os princípios do pensamento 
sistêmico tradicional (hard systems thinking) a situações problemáticas do dia-a-dia que envolvam aspectos humanos e, portanto, diferentes - e muitas vezes conflitantes - culturas, visões de mundo, pontos de vista e interesses. $\mathrm{O}$ hard systems thinking pressupõe que o mundo constitui-se de um conjunto de sistemas que podem ser observados objetivamente e que trabalham interligados, e que eventualmente alguns desses sistemas precisam ser redesenhados para funcionarem melhor.

.Já o pensamento sistêmico em que se baseia o SSM (soft systems thinking) pressupõe que o observador enxerga o mundo como algo complexo, que pode ser entendido e modelado de diversas formas, em um processo cíclico e baseado num sistema de aprendizado. Desse modo, a palavra sistema não seria mais empregada para definir o mundo (como fazemos inconscientemente no nosso cotidiano), e sim para definir o método de aprendizado que utilizamos para compreender esse mundo.

A experiência adquirida com os projetos de pesquisa-ação realizados por Checkland e seus colegas levaria eventualmente ao desenvolvimento da SSM como um processo composto por sete estágios, como mostra a figura abaixo:

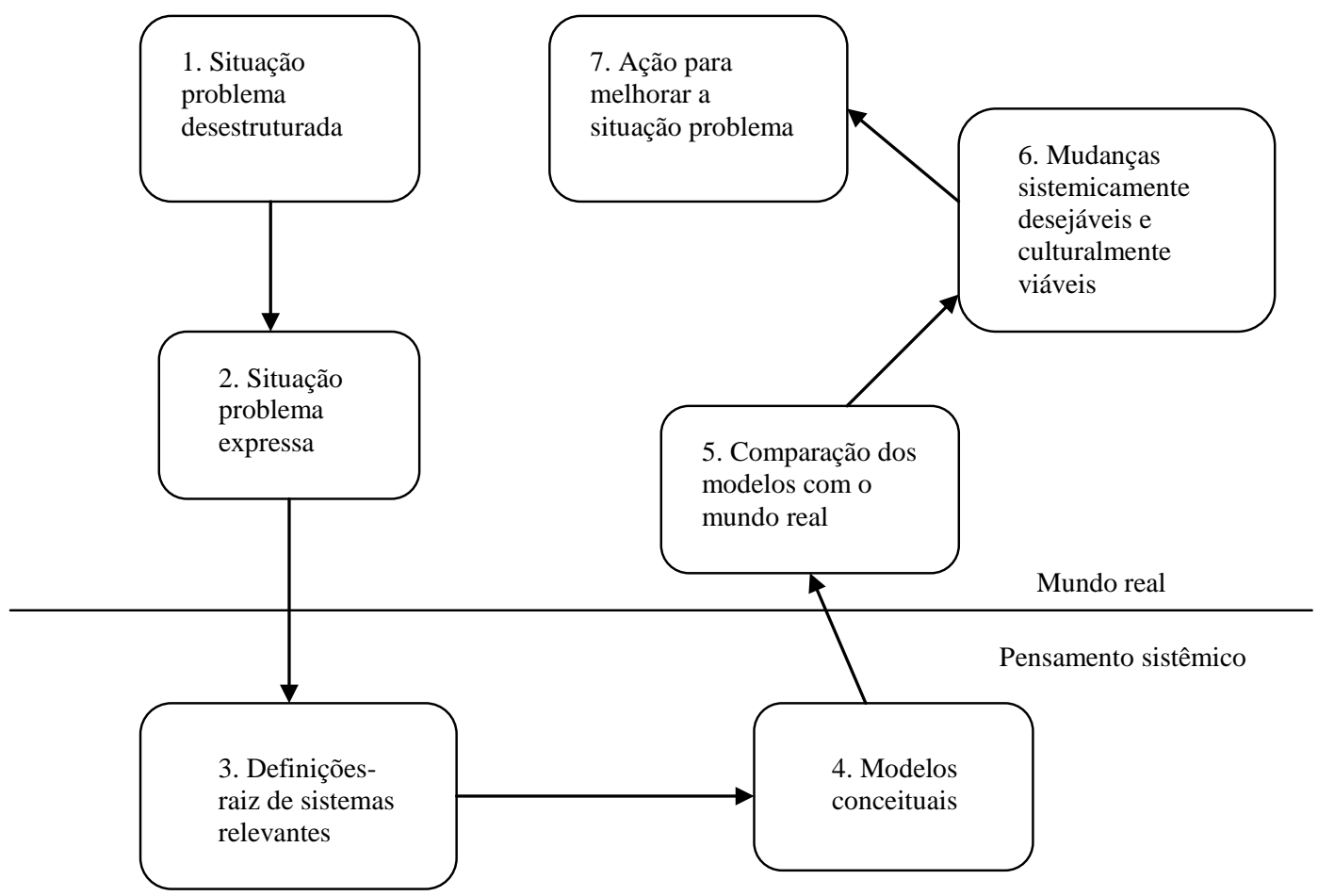

CHECKLAND $(1981,1999,2001)$ durante o desenvolvimento da SSM percebeu que o uso do mnemônico CATWOE poderia ser útil na definição e construção dos modelos relevantes. O CATWOE é composto pelos elementos que têm de estar no modelo relevante, como descrito a seguir:

$\square$ C - Client - Vítimas/Beneficiários de T

$\square$ A - Actors - Realizadores das atividades explicitadas em T

$\square \mathbf{T}$ - Transformation Process - Em que consiste a transformação T?

$\square$ W - Weltanschaungen - Que visão de mundo está por trás da definição da transformação T?

$\square$ O - Owner - Quem pode interromper a transformação T?

$\square$ E - Environmental Constraints - Quais as restrições admitidas no ambiente?

\section{O PROJETO PAPESCA}


A PAPESCA (Pesquisa-ação na Cadeia Produtiva da Pesca) é um projeto que engloba atividades de ensino, pesquisa e extensão e é desenvolvido pelo Núcleo de Solidariedade Técnica (SOLTEC/UFRJ), núcleo de pesquisa e extensão fundado em 13 de março de 2003 no âmbito do Departamento de Engenharia Industrial (DEI) da Escola Politécnica da UFRJ (POLI/UFRJ). O projeto foi iniciado em julho de 2004, inicialmente focalizado na cidade de Macaé (RJ), e hoje está sendo expandido para outras cidades do Norte do Estado do Rio de Janeiro.

A PAPESCA foi criada inicialmente com a intenção de identificar entraves à sustentabilidade da cadeia produtiva da pesca profissional artesanal na região e elaborar e participar da gestão de projetos de intervenção, sob uma perspectiva interdisciplinar e adotando a pesquisa-ação como proposta metodológica. O projeto apresentava um caráter sistêmico e integral, na medida em que observava a cadeia produtiva como um todo e não seus elos separadamente, sob a perspectiva do desenvolvimento local social e solidário da região e uma gestão responsável dos seus recursos naturais. O objetivo inicial do projeto era "contribuir para a sustentabilidade da cadeia produtiva da pesca e da Escola de Pescadores visando ao desenvolvimento local social e solidário de Macaé"(SOLTEC/UFRJ, 2006).

Entre 2004 e 2005 a equipe do projeto desenvolveu e facilitou um processo de diagnóstico participativo dos entraves à cadeia produtiva da pesca que envolveu a realização de entrevistas individuais, quatro reuniões coletivas e duas reuniões gerais, com a participação de 100 cidadãos (a maioria, pescadores e pescadoras) e 30 instituições (entidades da sociedade civil, colônia, associações de proteção ambiental e de pescadores, secretarias da prefeitura, ministérios, instituições acadêmicas e órgãos de fomento federais) (SOLTEC/UFRJ, 2009).

A partir de janeiro de 2008 a equipe da PAPESCA iniciou uma série de Seminários de Integração Metodológica, criados a partir da percepção da necessidade do redimensionamento da pesquisa, tanto no aspecto geográfico como no aspecto conceitual. Nesses Seminários discutiu-se a necessidade da incorporação, por parte da equipe, de conceitos como capital social, resiliência, ecodesenvolvimento, cadeia produtiva da pesca, gestão de recursos naturais, políticas públicas e consórcios públicos, bem como a criação da Rede Solidária da Pesca.

A Rede Solidária da Pesca, criada em 2006, visa facilitar a articulação entre projetos e instituições que tenham como objetivo a sustentabilidade da cadeia produtiva da pesca artesanal, com base nos princípios da Economia Solidária. A Rede busca constituir um debate sobre a questão da pesca no Brasil, ao mesmo tempo construindo uma visão nacional e reconhecendo a autonomia de ação dos projetos que a integram, de acordo com as especificidades sociais, econômicas, culturais e ambientais das regiões nas quais as intervenções são realizadas.

Durante os anos de 2008 e 2009 foram realizadas diversas oficinas e encontros visando à extensão da Rede e da PAPESCA/UFRJ para o Litoral Fluminense (RJ). Parte dessas ações se deve ao processo de interiorização da UFRJ.

Essas ações e articulações levaram à constatação de que a PAPESCA/UFRJ deve atuar dentro de uma perspectiva interdisciplinar, inter e intrainstitucional, e se redefinir como um projeto de pesquisa em rede, aberto ao diálogo e à atuação conjunta com outros pesquisadores e centros de pesquisa com os quais compartilhe seus objetivos ambientais e sociais. Dentro desse quadro o objetivo geral da PAPESCA/UFRJ também é reconfigurado, e passa a ser o de "contribuir com o desenvolvimento da gestão compartilhada da pesca artesanal e aqüicultura familiar no litoral fluminense”. (SOLTEC/UFRJ, 2009). 


\section{APLICAÇÃO DA SOFT SYSTEMS METHODOLOGY}

Nessa seção será mostrado um exemplo de aplicação da Soft Systems Methodology (SSM) ao projeto PAPESCA. Mais especificamente, serão abordadas as fases de descrição da definição-raiz e dos elementos que compõem o CATWOE e de construção de modelo(s) relevante(s) compostos por atividades com propósito (estágios 3 e 4 do modelo de sete etapas da SSM).

Essas são possíveis perspectivas para o projeto PAPESCA:

$\square$ Um meio para melhorar as condições de vida e trabalho dos pescadores artesanais de Macaé;

$\square$ Um meio para melhorar as condições de vida e trabalho dos construtores de barcos de Macaé;

$\square$ Preservar os ecossistemas marinhos da região;

$\square$ Identificar os entraves à cadeia produtiva da pesca em Macaé;

$\square$ Desenvolver a consciência ambiental dos trabalhadores da pesca;

$\square$ Proporcionar aos estudantes que fazem parte da equipe do SOLTEC/UFRJ um contato com problemas concretos da vida real por meio de um projeto de extensão e assim, aprimorar suas formações;

$\square$ Facilitar a interação entre os diversos atores sociais envolvidos na problemática da pesca em Macaé.

\subsection{Perspectiva um}

Considerando-se a primeira perspectiva ("um meio para melhorar as condições de vida e trabalho dos pescadores artesanais de Macaé”), para o modelo relevante correspondente a ela poder-se-ia considerar a seguinte definição-raiz:

"Um sistema controlado pelo SOLTEC/UFRJ no qual uma equipe de pesquisadores busca melhorar as condições de vida e trabalho dos pescadores artesanais de Macaé por meio de uma metodologia participativa que possibilite à equipe diagnosticar os problemas, encontrar soluções e ajudar a implementá-las”

O CATWOE desse sistema seria:

$\square$ Clients - Pescadores artesanais de Macaé

$\square$ Actors - Pesquisadores do SOLTEC/UFRJ envolvidos no projeto;

$\square$ Transformation - Péssimas condições de vida e trabalho dos pescadores $\rightarrow$ Melhores condições de vida e trabalho dos pescadores;

$\square$ Weltanschaungen - É inaceitável que pessoas (como os trabalhadores da pesca de Macaé) convivam com péssimas condições de vida;

$\square$ Owners - Coordenação do SOLTEC/UFRJ;

$\square$ Environmental Constraints - Metodologias participativas disponíveis; prédisposição dos pescadores em trabalhar em conjunto com os pesquisadores; características pessoais dos pesquisadores que os capacitem, ou não, a conduzirem um processo de intervenção baseado em uma metodologia participativa.

A figura abaixo mostra o modelo relevante dessa perspectiva. 


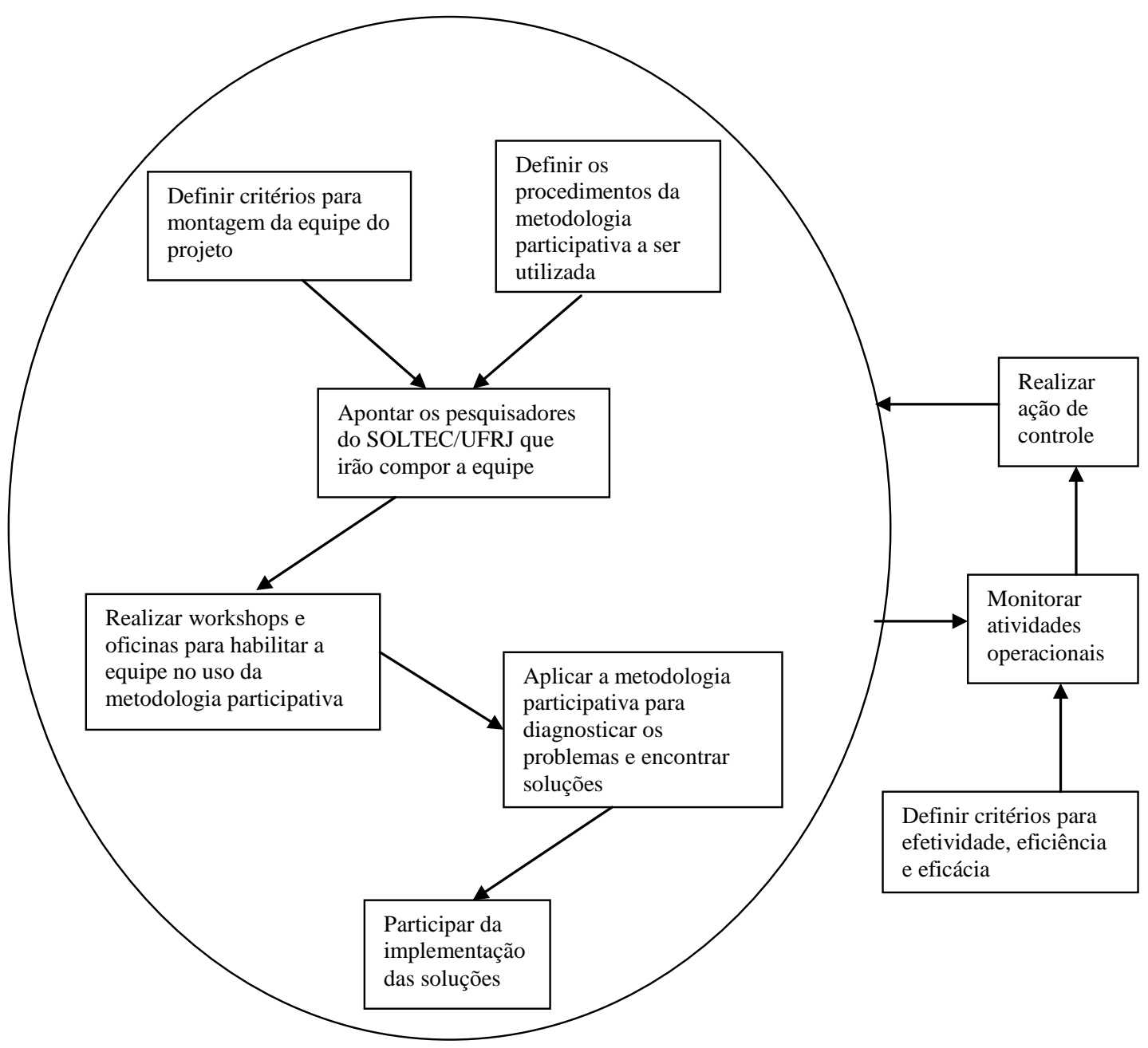

CHECKLAND (1999) afirma que se deve, inicialmente, construir modelos com baixo "nível de resolução" (com pequeno detalhamento) e depois considerar cada atividade como um subsistema, com definição-raiz e atividades próprias. Seguindo essa orientação, a atividade "aplicar a metodologia participativa para diagnosticar os problemas e encontrar soluções" pode ser mais bem detalhada se for tomada como um subsistema do sistema descrito acima.

A definição-raiz deste subsistema poderia ser:

"Um sistema controlado pela coordenação do SOLTEC/UFRJ que se utilize de uma metodologia participativa, baseada na constante interação entre pesquisadores e atores sociais, para diagnosticar os problemas e encontrar soluções para os problemas enfrentados pelos pescadores de Macaé”.

O CATWOE correspondente a essa definição-raiz seria:

$\square$ Clients - pescadores de Macaé

$\square$ Actors - equipe de pesquisadores do SOLTEC/UFRJ, atores sociais envolvidos com a pesca em Macaé

$\square$ Transformation - Inexistência de diagnóstico de problemas e formulação de soluções $\rightarrow$ Problemas e possíveis soluções identificadas por um processo participativo

$\square$ Weltanschaungen - Um processo participativo implica os atores direta ou indiretamente envolvidos no diagnóstico de problemas e busca de soluções, o que os motiva e aumenta a possibilidade de êxito da pesquisa 
$\square$ Owners - A coordenação do SOLTEC/UFRJ

$\square$ Environmental Constraints - Disponibilidade e interesse dos agentes sociais em participar de um projeto de intervenção de natureza participativa, conflitos pessoais entre os agentes, capacidade dos pesquisadores em atuar como facilitadores

A figura abaixo mostra o modelo relevante desse subsistema.

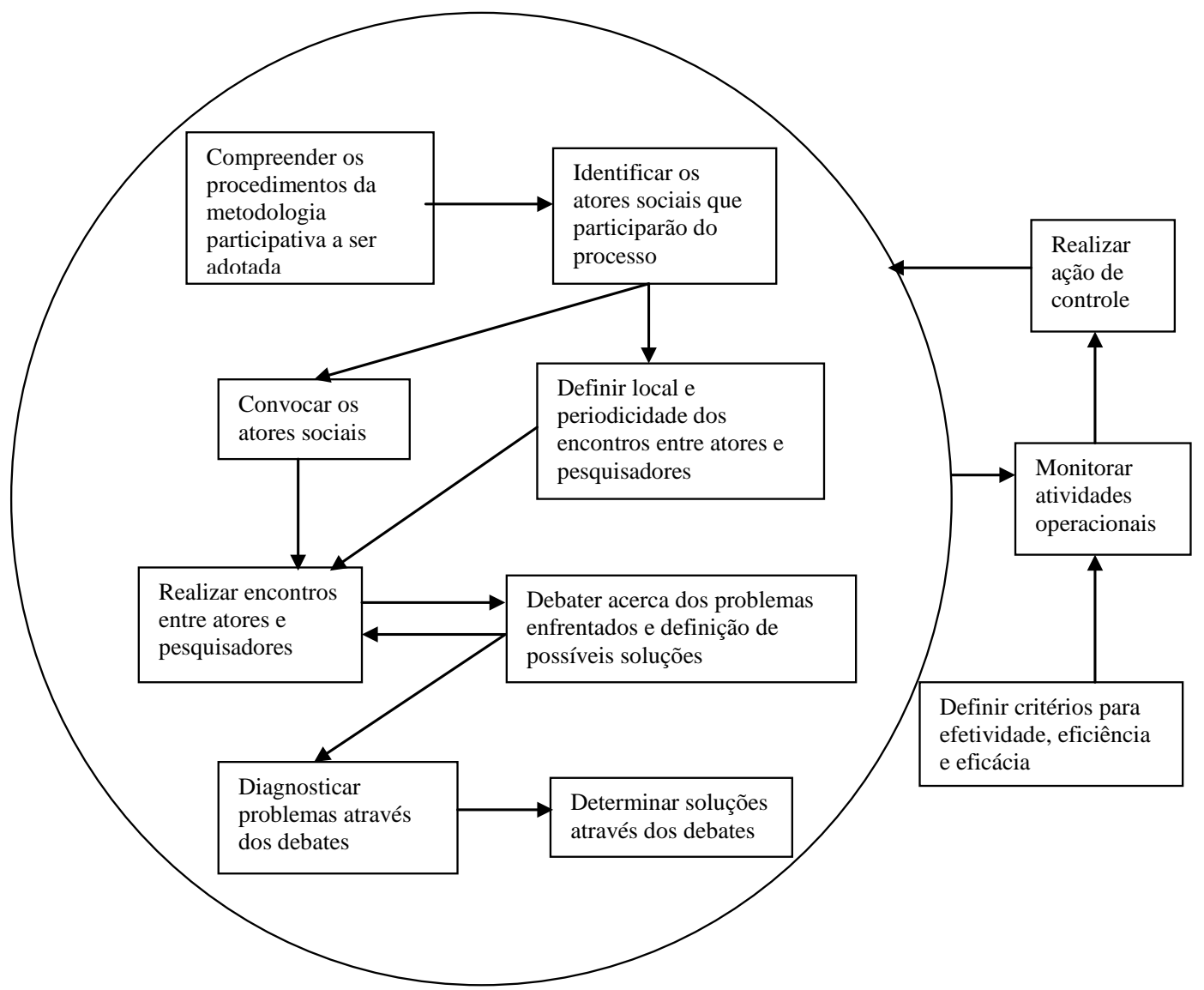

\subsection{PERSPECTIVA DOIS}

Uma das maiores potencialidades da SSM é permitir que sejam examinadas várias perspectivas do objeto de estudo (uma situação problemática, ou como no caso deste trabalho, um projeto) visando compreendê-lo da maneira mais ampla possível. Assim, iremos desenvolver o modelo relevante da perspectiva "proporcionar aos estudantes que fazem parte da equipe do SOLTEC/UFRJ um contato com problemas concretos da vida real por meio de um projeto de extensão e assim, aprimorar suas formações”. A definição-raiz para essa perspectiva poderia ser:

"Um sistema controlado pela coordenação do SOLTEC/UFRJ, financiado por órgãos de fomento, que permita que alunos da graduação participem de um projeto de extensão que lhes permita ter contato com problemas da vida real e assim, aprimorar suas formações e neles despertar uma consciência cidadã"

O CATWOE correspondente:

$\square$ Clients - alunos da graduação

$\square$ Actors - Órgãos de fomento à extensão universitária

$\square$ Transformation - Alunos com formação apenas teórica $\rightarrow$ Alunos com a formação enriquecida pelo contato com a realidade

$\square$ Weltanschaungen - Participar de projetos de extensão é fundamental para a 
formação dos alunos

$\square$ Owner - A coordenação do SOLTEC/UFRJ

$\square$ Environmental Constraints - Existência de programas de fomento à extensão; percentual dos recursos oriundos desses programas disponível para o SOLTEC/UFRJ; carga horária dos alunos que possibilite sua participação em projetos de extensão

A figura abaixo mostra o modelo relevante desse sistema.

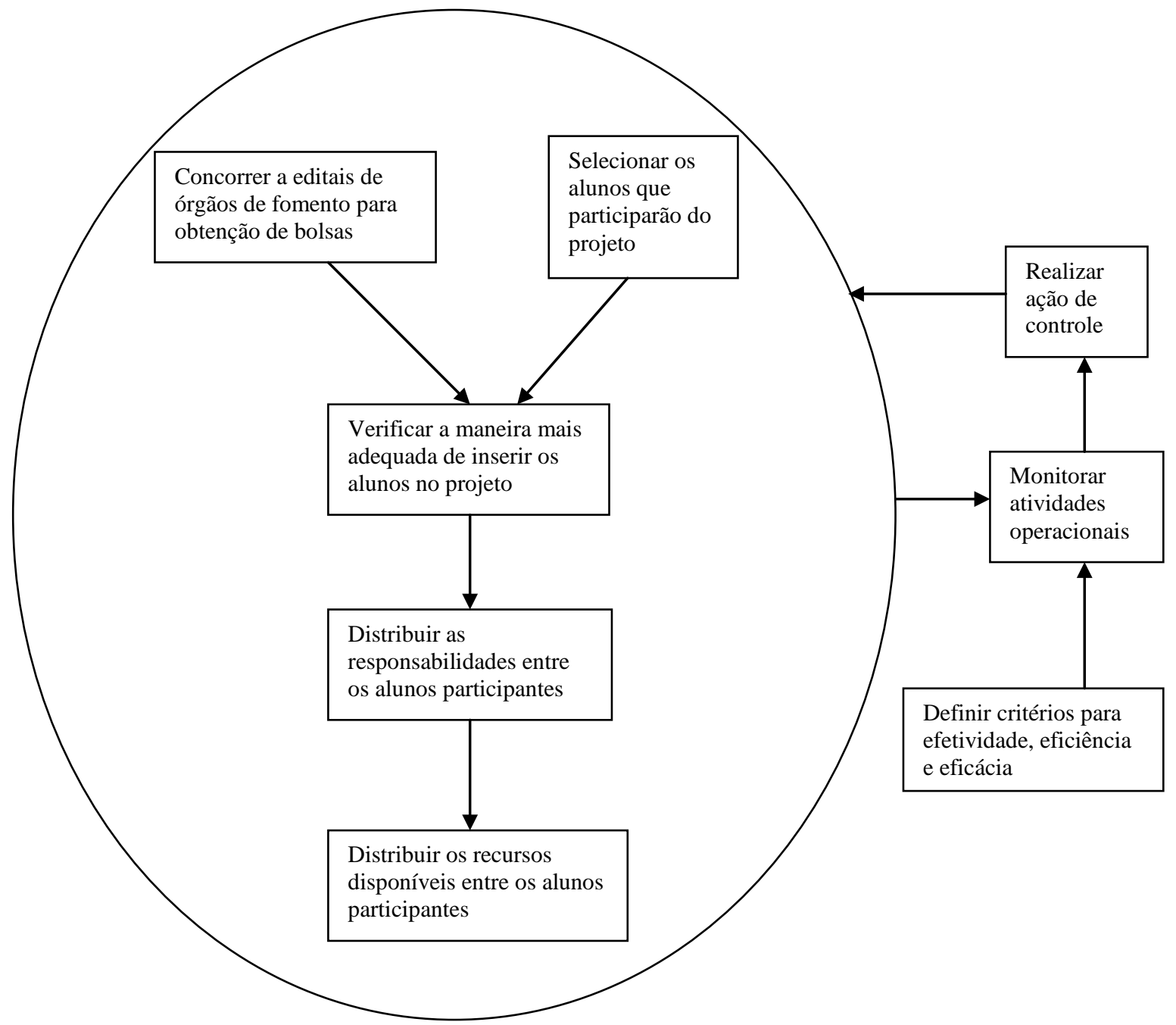

A utilidade da aplicação da SSM é a de permitir que se tenham diferentes perspectivas do objeto de estudo (em geral, uma situação-problema, mas que também pode ser, como no caso deste trabalho, um projeto ou um programa) e assim, obter uma melhor e mais ampla compreensão deste. A utilização da SSM proporcionaria ainda a identificação de várias perspectivas na organização do projeto de extensão (i.e. as atividades necessárias para a consecução de seus objetivos), englobando a visão de todos os participantes e não apenas as dos gestores.

\section{CONSIDERAÇÕES FINAIS}

Nas últimas duas décadas a Extensão Universitária no Brasil experimentou um grande salto, não apenas com relação ao aumento do número de programas e projetos de extensão, fruto do crescimento da importância das Pró-Reitorias de Extensão nas Universidades e dos programas governamentais de incentivo, mas também no que se refere às práticas desenvolvidas e à própria conceituação da extensão. 
Como salienta REIS (1996), a extensão no Brasil evoluiu de uma linha eventistainorgânica, nas quais as ações (materializadas primordialmente na forma de oferecimento de cursos e prestação de serviços) eram isoladas e desvinculadas das outras atividades-fim das Universidades (o ensino e a produção de conhecimento) para uma linha processual-orgânica, na qual as atividades extensionistas ganham caráter permanente, "imbricados ou inerentes ao processo formativo (ensino) e à produção de conhecimento (pesquisa) da universidade, em parceria político-pedagógica com a sociedade civil ou política, numa dimensão mutuamente oxigenante e mutuamente transformante”.

Com relação às práticas, vem igualmente se verificando um aumento da utilização de métodos participativos em projetos de Extensão Universitária.

Diante deste cenário, em que a extensão universitária torna-se parte indispensável da formação dos futuros profissionais, o presente trabalho exemplificou uma aplicação da Soft Systems Methodology a um projeto de extensão, o PAPESCA/UFRJ (mais especificamente, foram aplicadas as etapas 3 e 4 do modelo de sete estágios).

Em projetos como o PAPESCA/UFRJ (voltado para temas como o Desenvolvimento Local de regiões e gestão compartilhada de recursos naturais) é preciso criar condições para que se instale um debate aberto no qual os diversos agentes sociais possam expressar seus pontos de vista quanto à situação-problema, e que facilite a obtenção de soluções que levem ao comprometimento de todos para a ação.

A Soft Systems Methodology propicia o estabelecimento desse debate, por seu caráter eminentemente participativo, além de se tratar de uma metodologia valiosa para a exploração e entendimento de uma situação, e/ou organização e avaliação de um projeto. Como foi demonstrado neste trabalho, a SSM permite que emirjam diferentes perspectivas do objeto de estudo. No caso da avaliação e/ou organização de um projeto, a metodologia permite ainda que sejam considerados os pontos de vista de todos os interessados, e não apenas os dos gestores.

Com relação a futuras pesquisas similares, sugere-se o emprego de outros MEPs, tais como Strategic Options Development and Analysis (SODA), Strategic Choice Approach (SCA), Análise de Robustez, Drama Theory ou uma mescla de diversas abordagens (multimetodologia).

\section{REFERÊNCIAS BIBLIOGRÁFICAS}

[1] CHECKLAND, P. (1972). Towards a systems-based methodology for real-world problem solving. Journal of Systems Engineering, 3(2), 87-116.

[2] CHECKLAND, P. (1981). Systems Thinking, Systems Practice. John Wiley \& Sons, Ltd., Chichester.

[3] CHECKLAND, P. (2001). Soft Systems Methodology. In: Rational Analysis for a Problematic World Revisited. Rosenhead, J.\& Mingers, J. (Orgs), John Wiley \& Sons, Ltd., 61-89.

[4] CHECKLAND, P. (1999). Soft Systems Methodology: a 30-year Retrospective. John Wiley \& Sons, Ltd., Chichester.

[5] CHECKLAND, P. \& POULTER, J. (1994). Varieties of systems thinking: The case of soft systems methodology, System Dynamics Review, 10, 189-197.

[6] CHECKLAND, P. \& POULTER, J. (2006) Learning for action: a Short Definitive Account of Soft Systems Methodology and Its Use for Practitioners, Teachers and Students. John Wiley \& Sons, Ltd., Chichester. 
[7] CHECKLAND, P. \& SCHOLES, J.(1990). Soft Systems Methodology in Action. John Wiley \& Sons, Ltd., Chichester.

[8] PLANO NACIONAL DE EXTENSÃO UNIVERSITÁRIA (1999). Brasil. Disponível em: $<$ http://www.renex.org.br/index.php?option=com_content\&view=article\&id=45\&It emid=20>. Acesso em: 13 de agosto de 2013.

[9] REIS, R.H., (1996). Histórico, Tipologias e Proposições Sobre a Extensão Universitária no Brasil. Linhas Críticas, 2(2), 41-47.

[10] ROSENHEAD, J. (1996). What's the problem? An introduction to problem structuring methods. Interfaces, 126(6),117-131.

[11] ROSENHEAD, J. \& MINGERS, J. (2001). Rational Analysis for a Problematic World Revisited. John Wiley \& Sons, Ltd., Chichester.

[12] SOLTEC/UFRJ. (2006). Pesquisa-Ação na Cadeia Produtiva da Pesca em Macaé PAPESCA/MACAÉ - Relatório Final. Brasil. Disponível em <http://papescalitoralfluminense.blogspot.com/> Acesso em: 13 de março de 2014.

[13] SOLTEC/UFRJ. (2009). Relatório Analítico da Pesquisa Ação na Cadeia Produtiva da Pesca PAPESCA/UFRJ - 2004-2008. Brasil. Disponível em <http://papescalitoralfluminense.blogspot.com/.> Acesso em: 13 de março de 2014.

[14] THIOLLENT, M.J. (2008). Avanços da metodologia e da participação na extensão universitária. In: Metodologia para Projetos de Extensão: Apresentação e Discussão. Filho, T.A.\&Thiollent, M.J. (Orgs). Cubo Multimídia, 1-7. 